\title{
List of Cancer Cell Codes
}

This set of codes for 438 cancer cells, named as CCC codes, are defined and tried out in the books by the author. The codes of some normal cells are also listed below.

$293 T$

3T3-L1

5637

786-0

9KB

A-10

A2058

A278

A2780

A2780CisR

A2780/DDP

A2780/Tax

A375

A375-S2

A431

A498

A549

A549 NSCL

A549/ATCC

ACC-MESO-1

ACHN

AGS

AsPC-1

B16

B16F1

B16-F-10

BC

$B C-1$

BCA-1

BEAS2B

Bel7402

BG02

BGC823

BOWES

BR1

BSC

BSC-1

BSY1

BT-483

BT549

BT-549

BXF-1218L

BXF-T24

BXPC kidney epithelial cells

murine fibroblasts

superficial bladder cancer (cell)

hmn renal cancer (cell)

$\mathrm{hmn}$ epidermatoid nasopharyngeal carcinoma (cell)

rat aorta cells

hmn (cell)

hmn ovarian tumor (cell)

hmn ovarian tumor (cell)

hmn ovarian tumor (cell)

hmn ovarian tumor (cell)

hmn ovarian tumor (cell)

hmn melanoma (cell)

hmn melanoma (cell)

hmn epidermic cancer (cell)

hmn renal cancer (cell)

hmn nonsmall cell lung cancer (cell)

hmn nonsmall cell lung cancer (cell)

hmn nonsmall cell lung cancer (cell)

hmn malignant pleural mesothelioma (cell)

hmn renal cancer (cell)

gastric adenocarcinoma (cell)

hmn pancreatic cancer (cell)

mouse melanoma (cell)

mouse melanoma (cell)

mouse melanoma (cell)

hmn breast cancer (cell)

hmn breast cancer (cell)

hmn breast cancer (cell)

normal hmn lung bronchial cells

hmn liver cancer (cell)

normal hmn embryonic stem cells

hmn gastric cancer (cell)

hmn cells

DNA repair competent Chinese hamster ovary (cell)

normal monkey kidney cells

normal African Green Monkey kidney cells

breast cancer (cell)

hmn breast carcinoma (cell)

hmn galactophore cancer (cell)

hmn breast cancer (cell)

hmn bladder cancer (cell)

hmn bladder cancer (cell)

hmn pancreas cancer (cell) 


\begin{tabular}{|c|c|}
\hline BXPC3 & hmn pancreas cancer (cell) \\
\hline C6 & rat glioma (cell) \\
\hline C26 & hmn colon carcinoma (cell) \\
\hline C38 & murine colon adenocarcinoma (cell) \\
\hline CA46 & hmn Burkitt’s lymphoma (cell) \\
\hline Ca9-22 & hmn gingival carcinoma (cell) \\
\hline $\mathrm{CaCo-2}$ & hmn epithelial colorectal adenocarcinoma (cell) \\
\hline CAKI-1 & hmn renal cancer (cell) \\
\hline Calu & prostate carcinoma (cell) \\
\hline Calu3 & nonsmall cell lung cancer (cell) \\
\hline CCRF-CEM & hmn T-cell acute lymphoblastic leukemia (cell) \\
\hline CCRF-CEMT & leukemia (cell) \\
\hline CEM & hmn leukemia (cell) \\
\hline CEM-TART & T cells that express both HIV-1 tat and rev \\
\hline CFU-GM & $\mathrm{hmn} /$ murine hematopoietic progenitor cells \\
\hline $\mathrm{CHO}$ & Chinese hamster ovary cells \\
\hline $\mathrm{CHO}-\mathrm{K} 1$ & subclone of normal Chinese hamster ovary cells \\
\hline CML K562 & chronic myelogenous leukemia (cell) \\
\hline CNE & hmn nasopharyngeal carcinoma (cell) \\
\hline CNE2 & hmn nasopharyngeal carcinoma (cell) \\
\hline CNS SF295 & hmn brain tumor (cell) \\
\hline CNXF-498NL & hmn glioblastoma cancer (cell) \\
\hline CNXF-SF268 & hmn glioblastoma cancer (cell) \\
\hline Colo320 & hmn colorectal cancer (cell) \\
\hline Colo357 & hmn colorectal cancer (cell) \\
\hline Colon26 & colorectal cancer (cell) \\
\hline Colon38 & mus colorectal cancer (cell) \\
\hline Colon205 & colorectal cancer (cell) \\
\hline Colon250 & colorectal cancer (cell) \\
\hline CV-1 & monkey kidney fibroblasts \\
\hline CXF-HCT116 & hmn colon cancer (cell) \\
\hline CXF-HT29 & hmn colon cancer (cell) \\
\hline DAMB & hmn mammary carcinoma (cell) \\
\hline DG-75 & hmn B lymphocyte (cell) \\
\hline DLAT & Dalton's lymphoma ascites tumor (cell) \\
\hline DLD-1 & hmn colorectal adenocarcinoma (cell) \\
\hline DLDH & hmn colorectal adenocarcinoma (cell) \\
\hline DMS114 & hmn lung cancer (cell) \\
\hline DMS273 & hmn small cell lung cancer (cell) \\
\hline Doay & hmn medulloblastoma (cell) \\
\hline Dox40 & hmn myeloma (cell) \\
\hline DU145 & prostate cancer (cell) \\
\hline DU4475 & breast cancer (cell) \\
\hline E39 & hmn renal carcinoma (cell) \\
\hline EAC & Ehrlich ascites carcinoma (cell) \\
\hline EKVX & hmn nonsmall cell lung cancer (cell) \\
\hline EM9 & topoisomerase I-sensitive Chinese hamster ovary (cell) \\
\hline EMT-6 & mouse tumor cells \\
\hline EPC & carp epithelium (cell) \\
\hline
\end{tabular}


EVLC-2

F1

FADU

Farage

Fem-X

$\mathrm{Fl}$

FM3C

G402

GM7373

GR-III

GXF-251L

H116

H125

H441

H460

H522

H1299

H1325

H1975

H2122

H2887

H69AR

H929

H9c2

HBC 4

$\mathrm{HBC5}$

HBL100

HCC366

HCC2998

HCC-S102

$\mathrm{HCT}$

HCT8

HCT15

HCT29

HCT116

HCT116/mdr+

HCT116/topo

HCT116/VM46

HEK-293

HEL

HeLa

HeLa-APL

HeLa-S3

Hep2

Hер3B

HepA

Hepa1c1c7

HepG

HepG2
SV40 large T-antigen immortalized hmn umbilical vein cells

hmn amniotic epithelial cells

pharynx-sq cancer (cell)

hmn lymphoma (cell)

melanoma (cell)

hmn amniotic epithelial cell line

mus mammary tumor (cell)

hmn renal leiomyoblastoma

bovine endothelial (cell)

adenocarcinoma (cell)

hmn stomach cancer (cell)

hmn colorectal cancer (cell)

hmn colorectal cancer (cell)

hmn lung adenocarcinoma (cell)

hmn lung cancer (cell)

hmn nonsmall cell lung cancer (cell)

hmn lung adenocarcinoma (cell)

hmn nonsmall cell lung cancer (cell)

hmn cancer (cell)

hmn nonsmall cell lung cancer (cell)

hmn nonsmall cell lung cancer (cell)

multidrug-resistant small cell lung cancer (cell)

hmn myeloma (cell)

rat cardiac myoblasts

breast cancer (cell)

breast cancer (cell)

breast cancer (cell)

hmn nonsmall cell lung cancer (cell)

hmn colorectal cancer (cell)

hepatocellular carcinoma (cell)

hmn colorectal cancer (cell)

hmn colorectal cancer (cell)

hmn colorectal cancer (cell)

$\mathrm{hmn}$ colon adenocarcinoma (cell)

hmn colorectal cancer (cell)

overexpress $\mathrm{mdr}+\mathrm{hmn}$ colorectal cancer (cell)

resistant to etoposide hmn colorectal cancer (cell)

multidrug-resistant colorectal cancer (cell)

normal hmn epithelial kidney cells

hmn embryonic lung fibrocytes

hmn cervical epithelial carcinoma (cell)

hmn cervical epithelial cancer (cell)

hmn cervical epithelial cancer (cell)

hmn liver carcinoma (cell)

hmn liver cancer (cell)

hmn liver cancer ascites (cell)

mus liver cancer (cell)

hmn liver cancer (cell)

hmn liver cancer (cell) 


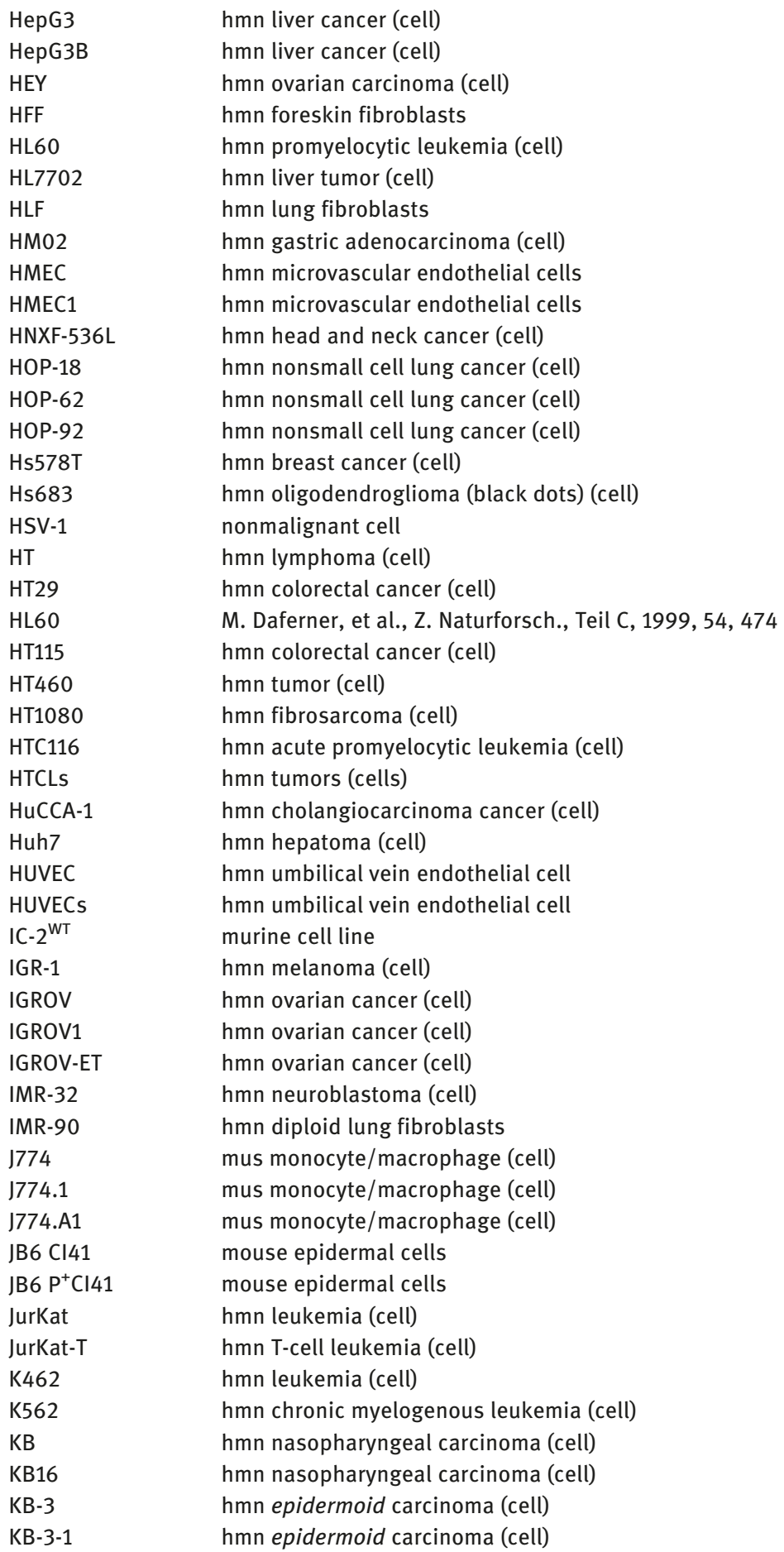




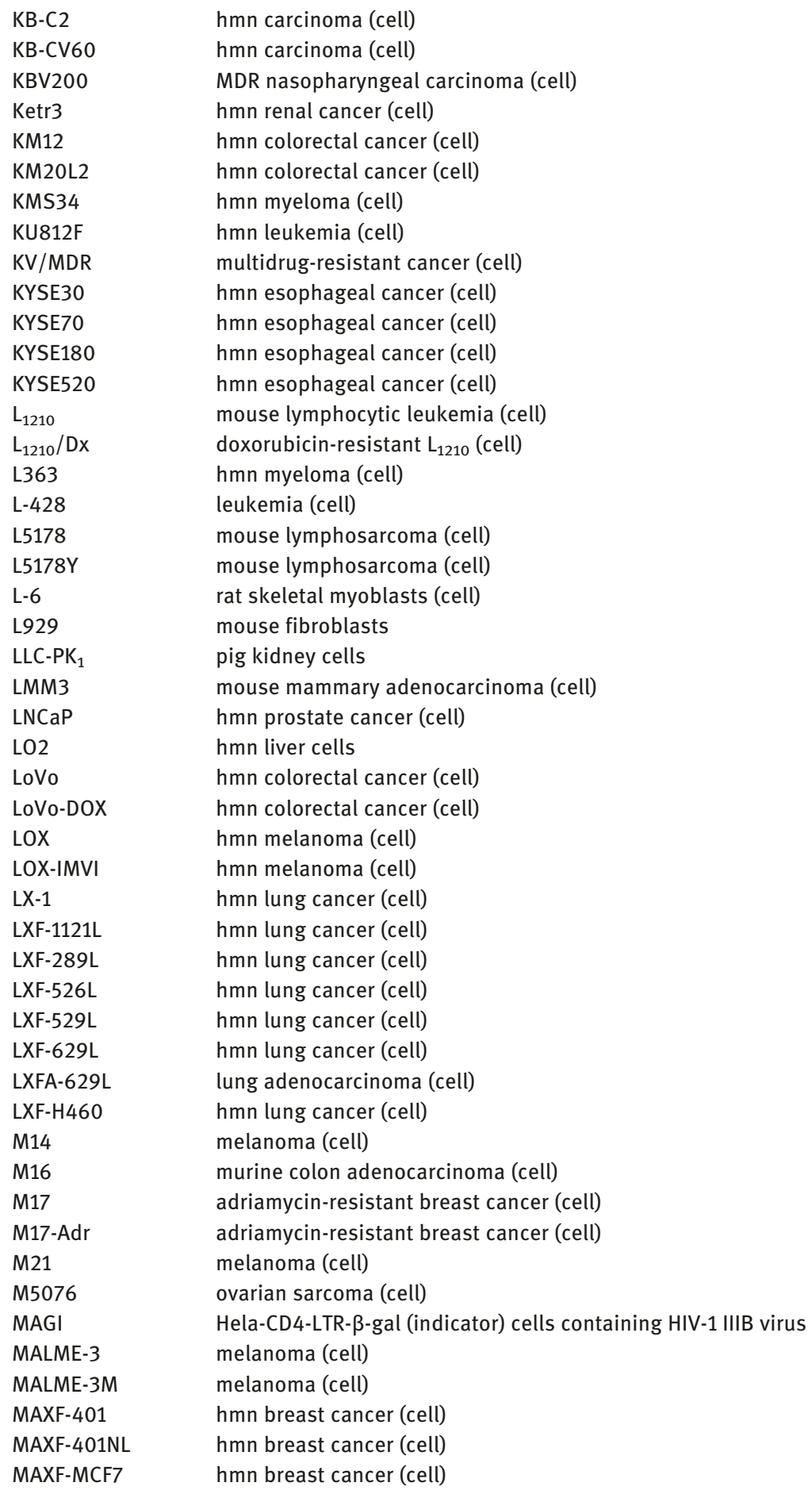




\begin{tabular}{|c|c|}
\hline MCF & hmn breast cancer (cell) \\
\hline MCF-10A & hmn breast epithelial (cell) \\
\hline MCF7 & hmn breast cancer (cell) \\
\hline MCF7 Adr & drug-resistant hmn breast MCF7 cancer (cell) \\
\hline MCF7/Adr & drug-resistant hmn breast MCF7 cancer (cell) \\
\hline MCF7/ADR-RES & drug-resistant hmn breast cancer MCF7 (cell) \\
\hline MCF12 & hmn esophageal cancer (cell) \\
\hline MDA231 & hmn breast cancer (cell) \\
\hline MDA361 & hmn breast cancer (cell) \\
\hline MDA435 & hmn breast cancer (cell) \\
\hline MDA468 & hmn breast cancer (cell) \\
\hline MDA-MB & hmn breast cancer (cell) \\
\hline MDA-MB-231 & hmn breast cancer (cell) \\
\hline MDA-MB-231/ATCC & hmn breast cancer (cell) \\
\hline MDA-MB-435 & hmn breast cancer (cell) \\
\hline MDA-MB-435s & hmn breast cancer (cell) \\
\hline MDA-MB-468 & hmn breast cancer (cell) \\
\hline MDA-N & hmn breast cancer (cell) \\
\hline MDCK & Madin-Darby canine (cell) \\
\hline ME180 & cervical cancer (cell) \\
\hline MEL28 & hmn melanoma (cell) \\
\hline MES-SA & hmn uterine (cell) \\
\hline MES-SA/DX5 & hmn uterine (cell) \\
\hline MEXF-276L & hmn melanoma (cell) \\
\hline MEXF-394NL & hmn melanoma (cell) \\
\hline MEXF-462NL & hmn melanoma (cell) \\
\hline MEXF-514L & hmn melanoma (cell) \\
\hline MEXF-520L & hmn melanoma (cell) \\
\hline MG63 & hmn osteosarcoma (cell) \\
\hline MGC-803 & hmn cancer (cell) \\
\hline $\mathrm{MiaPaCa}$ & hmn pancreas cancer (cell) \\
\hline Mia-PaCa-2 & hmn pancreas cancer (cell) \\
\hline MKN1 & hmn gastric cancer (cell) \\
\hline MKN7 & hmn gastric cancer (cell) \\
\hline MKN28 & hmn gastric cancer (cell) \\
\hline MKN45 & hmn gastric cancer (cell) \\
\hline MKN74 & hmn gastric cancer (cell) \\
\hline MM1S & hmn myeloma (cell) \\
\hline Molt3 & leukemia (cell) \\
\hline Molt4 & hmn T lymphocyte leukemia (cell) \\
\hline Mono-Mac-6 & mononuclear cells \\
\hline MPM ACC-MESO-1 & hmn malignant pleural mesothelioma \\
\hline MRC-5 & normal hmn diploid embryonic cells \\
\hline MRC5CV1 & SV40-transformed hmn fibroblasts \\
\hline MS-1 & mice endothelial cells \\
\hline MX-1 & hmn mammary carcinoma xenografts \\
\hline N18-RE-105 & neuronal hybridoma (cell) \\
\hline N18-T62 & mus neuroblastoma (cell) \\
\hline NAMALWA & leukemia (cell) \\
\hline
\end{tabular}




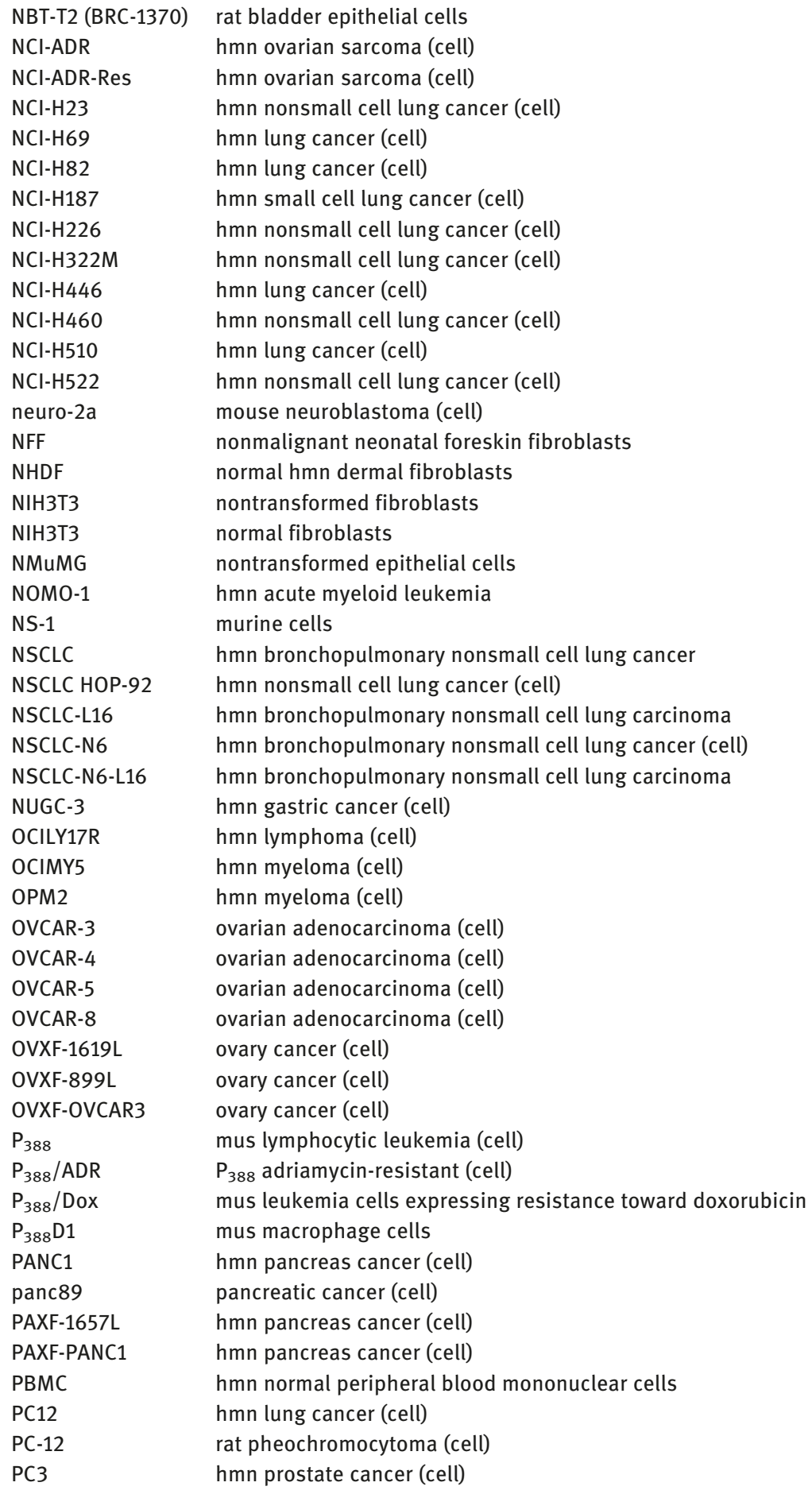




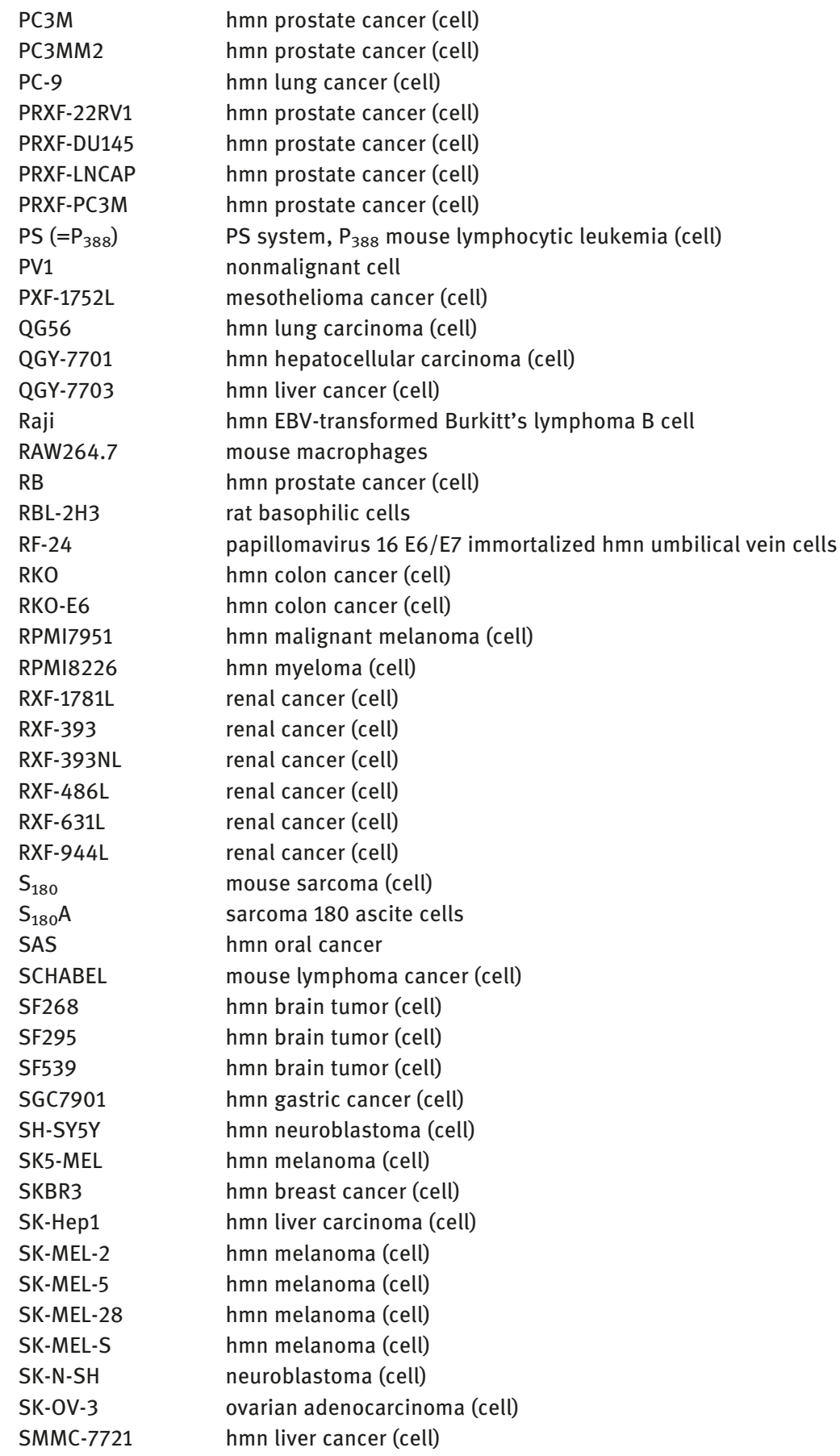




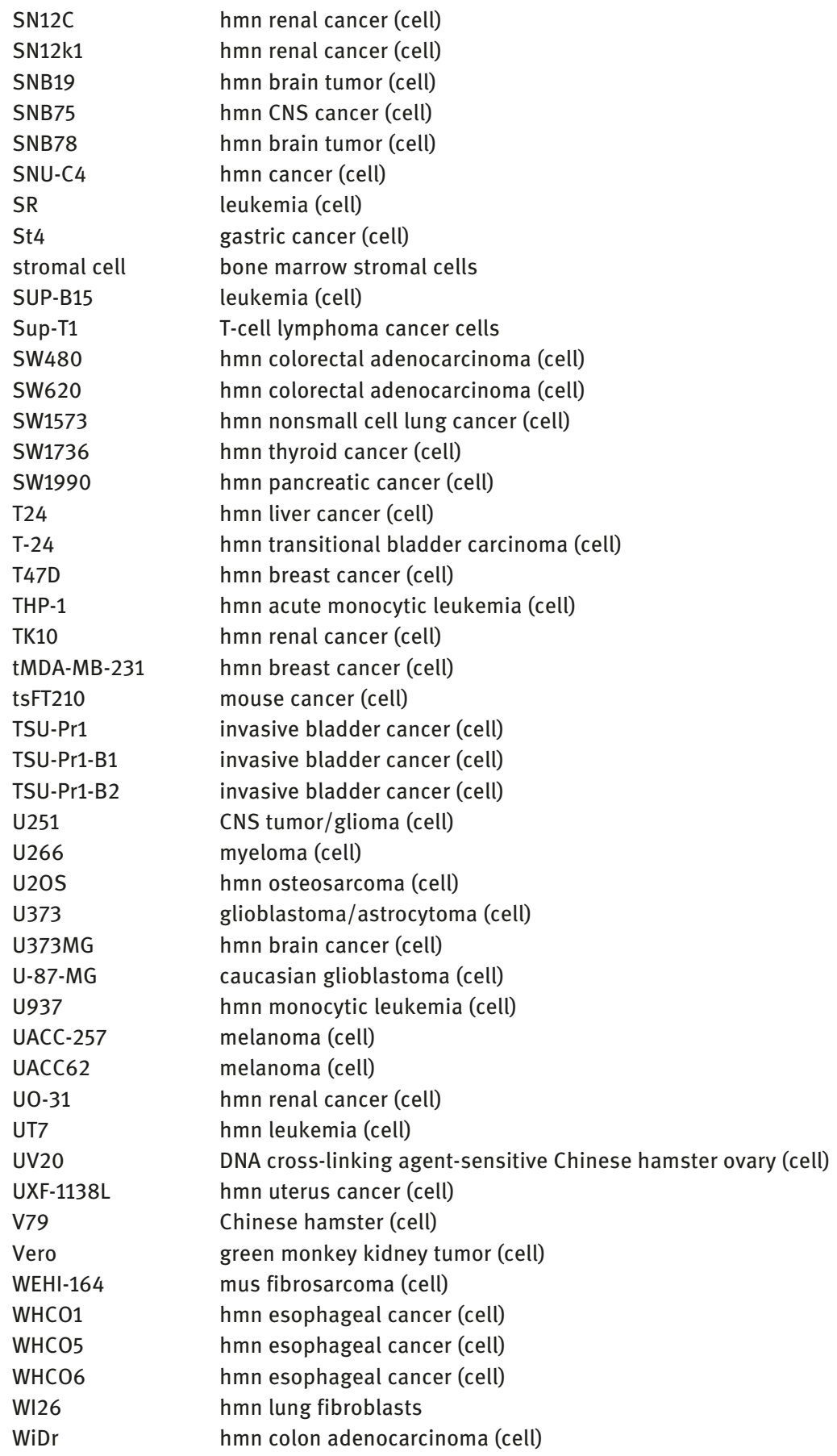


WMF

hmn prostate cancer (cell)

XF498

hmn CNS cancer (cell)

XRS-6

topoisomerase II-sensitive Chinese hamster ovary (cell)

$\mathrm{XVS}$

ZR-75-1 topoisomerase II-sensitive $\mathrm{CHO}$ cell hmn breast cancer (cell) 